\title{
Role of Diffusion-Weighted Magnetic Resonance Imaging in the Diagnosis of Hepatic Focal Lesions
}

\author{
Ahmed Okasha Mohammed ${ }^{1}$, Mohamed Mostafa Ali ${ }^{2}$, Hoda Rabea Hassan ${ }^{1}$, Wael Mohammed Wagdy*1 \\ ${ }^{1}$ Radiodiagnosis Department, ${ }^{2}$ Clinical Oncology Department, \\ Faculty of Medicine, South Valley University, Egypt \\ *Corresponding author: Wael Mohammed Wagdy, Mobile: (+20) 01005651501, Email: massa2000@yahoo.com
}

\begin{abstract}
Background: Diffusion-weighted imaging (DWI) is a novel imaging technique with a growing application in oncoimaging. This modality evaluates the diffusion of water molecules in various tissues, which is restricted in hypercellular regions such as malignant tissue. Apparent diffusion coefficient (ADC) is a method that can quantify the degree of restriction in tissues and can have diagnostic roles in the characterization of hepatic lesions.

Aim of the Work: To study the role of diffusion-weighted MRI sequence with quantitative ADC measurements as a useful tool in the differentiation between benign and malignant liver lesions.

Patients and Methods: The current study was carried out on thirty adult patients, 24 males (80\%), and 6 females (20\%). They were presented to Qena University Hospitals at the Radiodiagnosis Department.

Results: Both, qualitative evaluation of high b-value DW-MR images and quantitative evaluation of ADC maps are employed for lesion characterization. The ADC values of benign lesions are significantly higher than those of malignant lesions, with variable degrees of overlap between the pathological entities.

Conclusion: DW MR imaging has the potential to be a reasonable alternative technique to contrast-enhanced imaging.
\end{abstract}

Keywords: DWI, Hepatic focal lesions, ADC.

\section{INTRODUCTION}

Liver diseases have been known liver size and functions but the assessment of the exact pathology is grossly inadequate. Focal liver disease is a common diagnostic problem referred to the radiologists for evaluation owing to its nonspecific clinical presentation and marked inter-observer variation on clinical examination ${ }^{(\mathbf{1})}$.

The liver is considered a common site for many benign, primary malignant and metastatic focal lesions. Accurate detection and characterization of these tumors are crucial before treatment to ensure correct staging, to prevent tumors from being falsely rated as inoperable and patients with inoperable tumors from being scheduled for surgical procedures ${ }^{(2)}$.

Today, focal masses are diagnosed using ultrasonography (USG) and/or computed tomography (CT). Additionally, magnetic resonance imaging (MRI) is preferred when further characterization of these masses is needed ${ }^{(\mathbf{1})}$.

Ultrasound has a high sensitivity (Se) for FLLs detection but with low specificity (Sp) for discriminating between different entities. CT is recommended in patients with known malignancy for staging, and in patients with chronic liver disease or even in otherwise healthy persons for confirmation of a suspected liver tumor detected in the US. MRI is considered the imaging technique with the best Se and Sp for FLL diagnosis. Diffusion-weighted imaging (DWI) is a relatively recent non-contrast imaging tool that has a high contrast resolution allowing accurate FLLs detection and characterization ${ }^{(3)}$.

Diffusion-weighted imaging (DWI) has been reported to be useful for the early detection of small focal hepatic lesions. Moreover, DWI offers the possibility to obtain criteria for lesion characterization without the need for contrast agent administration by quantifying diffusion effects via apparent diffusion coefficient (ADC) measurements, with better results compared with those of conventional MR imaging ${ }^{(2)}$.

DWI could characterize specific tissue properties without any harm to patients, especially beneficial for those who are at risk for complications of a biopsy procedure ${ }^{(4)}$.

\section{AIM OF THE WORK}

The present work aimed to study the role of Diffusion-Weighted Magnetic Resonance Imaging (DW MRI) \& ADC in the detection of hepatic focal lesions and its ability to differentiate between benign and malignant hepatic focal lesions.

\section{PATIENTS AND METHODS}

The current study was performed in Qena University Hospitals, where thirty patients with hepatic focal lesions were included in this study. All patients underwent US before MR examination.

\section{Inclusion Criteria:}

1. Patients with hepatic focal lesions diagnosed by US or CT.

2. Hepatic focal lesion more than $10 \mathrm{~mm}$.

3. Age $>18$ years.

\section{Exclusion Criteria:}

1. Patients who have a heart pacemaker.

2. Patients who have a metallic foreign body. 
3. Young patients $<18$ years.

4. Hepatic focal lesion less than $10 \mathrm{~mm}$.

5. Patients with hepatic coma.

The patients were subjected to the following:

- Full clinical assessment including; recording of age, sex, and clinical presentation.

- Laboratory investigations \{Alpha fetoprotein\}.

- Abdominal MRI (pre- and post-contrast (Dynamic) study and diffusion-weighted imaging) done for all patients.

- The results were compared to the laboratory results, and other previous radiological findings and to the typical picture of lesions in dynamic MRI.

\section{Ethical approval:}

This study was conducted under the Helsinki's declarations, the study was approved by the Ethics Board of South Valley University and written informed consent was obtained from all patients.

\section{MR Examination:}

Conventional MRI, post-Gd- DTPA dynamic, and diffusion MR imaging studies were performed. First; characterization and detection of focal lesions were performed; second, the diffusion images with ADC values were reviewed. MR imaging was performed on a high field system (1.5 Tesla) magnet units (Philips Achieva) using a phased array coil to cover the whole liver.

\section{MR Protocol:}

\section{Pre-contrast imaging included:}

- T1 weighted (T1W) images: repetition time $\mathrm{TR}=10 \mathrm{msec}$, echo time $\mathrm{TE}=4.58 \mathrm{msec}$, matrix $179 \times 320$, slice thickness 7-8mm, slice gap 1- $2 \mathrm{~mm}$, and FOV $355 \mathrm{~mm}$.

- $\quad \mathrm{T} 2$ weighted (T2W) images (single shot free breathing): TR

- $\geq 445 \mathrm{msec}, \mathrm{TE}=26-28 \mathrm{msec}$, matrix (180-200)x240, slice thickness 7-8mm, slice gap 1-2mm, and FOV 365.

- T2 SPAIR (Spectral Attenuated Inversion Recovery) fat suppression sequence: $T R \geq 400 \mathrm{msec}, \mathrm{TE}=80 \mathrm{msec}$, matrix $204 \times 384$, slice thickness $7-8 \mathrm{~mm}$, slice gap 1$2 \mathrm{~mm}$ and FOV 365.

- In phase and out phase gradient echo sequence (Dual/FFE): TR= 75-100msec, $\mathrm{TE}=4.6 \mathrm{msec}$ for inphase and $2.3 \mathrm{msec}$ for out-phase, matrix $143 \times 240$, slice thickness 7-8mm, slice gap 0mm, and FOV 345.

\section{Dynamic study:}

This dynamic study was performed after bolus injection of $0.1 \mathrm{mmol} / \mathrm{kg}$ body weight of Gd-DTPA at a rate of $2 \mathrm{ml} / \mathrm{s}$, flushed with $20 \mathrm{ml}$ of sterile $0.9 \%$ saline solution through the antecubital vein. The injection of contrast media and saline solution was performed manually. Dynamic imaging using T1 THRIVE (HighResolution Isotropic Volume Examination) technique was performed in a triphasic way [arterial phase (16-20 sec.), Porto-venous phase (45-60 sec.) and delayed equilibrium phase (3-5 min.)] after administration of the contrast medium.

\section{Diffusion study:}

Respiratory-triggered fat-suppressed single-shot echoplanar DW imaging was performed in the transverse plane with tri-directional diffusion gradients by using $b$ values $(0,500 \& 1000) \mathrm{sec} / \mathrm{mm} 2$ to increase sensitivity to cellular packing. Parallel imaging with generalized auto-calibrating partially parallel acquisition (GRAPPA) with an acceleration factor of two was applied to improve image quality. The other parameters were as follows: repetition time $(\mathrm{TR}) \geq 1880$ msec, echo time (TE) $=70 \mathrm{msec}$, number of excitations $(\mathrm{NEX})=3$, matrix $256 \times 256$, slice thickness $7-8 \mathrm{~mm}$, slice gap $1-2 \mathrm{~mm}$, scan time 3-4min with a field of view as small as possible with $52 \%$ rectangular field of view.

\section{Imaging Evaluation:}

The morphological features of each lesion were recorded included size, shape, margin, and signal characteristics, the pattern of enhancement in the dynamic imaging as well as the number and size of the detected focal lesions. Then, the provisional diagnosis was reported. Second, we reviewed the diffusion images with ADC values for final radiological detection and characterization of focal lesions.

The results were compared to laboratory and other radiological findings (dynamic MRI) in all patients.

\section{ADC Calculation:}

The mean ADC of each detected focal lesion is measured by drawing a region of interest (ROI) over the lesion. The ADC was measured twice and the two measurements were averaged. To ensure that the same areas were measured, regions of interest were copied and pasted from DW images to ADC maps.

\section{Statistical analysis}

Recorded data were analyzed using the statistical package for social sciences, version 20.0 (SPSS Inc., Chicago, Illinois, USA). Quantitative data were expressed as mean \pm standard deviation (SD). Qualitative data were expressed as frequency and percentage. Independentsamples t-test of significance was used when comparing two means. Chi-square $\left(\mathrm{x}^{2}\right)$ test of significance was used to compare proportions between two qualitative parameters. The confidence interval was set to $95 \%$ and the margin of error accepted was set to $5 \%$. The p-value was considered significant as the following: $P$-value $<0.05$ was considered significant. Pvalue $<0.001$ was considered as highly significant $\&$ Pvalue $>0.05$ was considered insignificant.

\section{RESULTS}

The current study was carried out on thirty adult patients of both sex; all patients were presented to Qena university hospitals at the Radiodiagnosis Department. Thirty patients were included in this study, 24 males $(80 \%)$ and 6 females $(20 \%)$. 
Table (1): Numbers of lesions in cirrhotic and non-cirrhotic liver

\begin{tabular}{|c|c|c|}
\hline & Number of lesions & Percent \\
\hline Cirrhotic liver & 14 & $30 \%$ \\
\hline Non-cirrhotic liver & 32 & $70 \%$ \\
\hline
\end{tabular}

32 out of 46 lesions were found in non-cirrhotic liver, 17 of them was discovered by the abdominal US during the investigation for follow up of primary malignant, while the rest were accidental during abdominal U/S performed for abdominal pain or routine workup before the abdominal operation, while the other 14 lesions found in cirrhotic liver and were coming for follow up as in table (1). All cirrhotic patients were suffering from HCV.

Table (2): MRI sequences of the lesions.

\begin{tabular}{|c|c|c|c|c|c|}
\hline Lesion & T1 & T2 & Dynamic & DW & ADC \\
\hline CYST & hypo intense & hyper intense & Not enhanced & facilitated & $2.95+/-0.36 \times 10^{-3}$ \\
\hline HEMANGIOMA & hypo intense & hyper intense & Nodular enhanced & restricted & $2.2+/-0.16 \times 10^{-3}$ \\
\hline HCC & hyperintense & hyperintense & $\begin{array}{c}\text { Early enhanced, } \\
\text { early washout }\end{array}$ & restricted & $0.88+/-0.16 \times 10^{-3}$ \\
\hline METS & hypointense & hyperintense & $\begin{array}{c}\text { Early enhanced, } \\
\text { early washout }\end{array}$ & restricted & $1.03+/-0.12 \times 10^{-3}$ \\
\hline
\end{tabular}

In our study HCC show high signal on T1 WIs and high signal on T2 WIs and T2 FAT SAT WIs. On the post-contrast phases, the lesion showed mild enhancement in the arterial phase and early contrast washout in the subsequent phases with capsular enhancement in the delayed phase while mets show hypo-intense signals in T1 WIs and hyper-intense signals on T2 WIs.

Haemangioma shows hypo-intense signals in T1 WIs and hyperintense signals on T2 WIs and peripheral nodular enhancement in the dynamic phase. While cysts not enhanced in dynamic phase as demonstrated in Table (2).

\section{ADC values:}

- ADC values were obtained for all 46 focal hepatic lesions detected at consensus reading.

- The mean ADC value of the 15 benign lesions was $2.53+/-0.36$

- $\times 10-3 \mathrm{~mm} 2 / \mathrm{sec}$. ADC values of benign lesions were between $1.8+/-0.36 \times 10-3$ and $3.3+/-$ $0.36 \times 10-3 \mathrm{~mm} 2 / \mathrm{sec}$. The highest mean ADC value was for simple cysts.

- The ADC values of the 31 malignant lesions were between 0.50 and $1.3+/-0.16 \times 10-3 \mathrm{~mm} 2 / \mathrm{sec}$, with a mean value of $0.94+/-0.16 \times 10-3 \mathrm{~mm} 2 / \mathrm{sec}$ Among the malignant lesions, the lowest mean $\mathrm{ADC}$ value was for breast metastasis; while some HCC focal lesion was the highest mean ADC value.

- The difference between the mean ADC values of benign and malignant lesions was statistically significant $(\mathrm{P}<0.0001)$.

- No statistically significant differences in ADC values among the different benign lesions or the different malignant lesions.

Table (3): Mean ADC value of the different lesions.

\begin{tabular}{|l|l|}
\hline Lesion type & $\begin{array}{c}\text { Mean } \mathbf{A D C} \text { value } \\
\left(\mathbf{m m}^{2} / \mathbf{s e c}\right)\end{array}$ \\
\hline HCC & $0.88+/-0.16 \times 10^{-3}$ \\
\hline Cyst & $2.95+/-0.36 \times 10^{-3}$ \\
\hline Hemangioma & $2.2+/-0.16 \times 10^{-3}$ \\
\hline Metastasis & $1.03+/-0.12 \times 10^{-3}$ \\
\hline
\end{tabular}

Clinical history: A 55-year-old male patient presented with right hypochondrial pain and yellowish discoloration of the sclera. On US examination: The liver was cirrhotic with evidence of a hyperechoic right hepatic lobe focal lesion.

\section{Laboratory investigations: AFP: $500 \mathrm{ng}$}

On MR examination: The liver appeared cirrhotic and the right hepatic lobe was the seat of a small focal lesion at segment VIII, eliciting high signal on T1 WIs, and high signal on T2 WIs and T2 FAT SAT WIs. On the post-contrast phases, the lesion showed mild enhancement in the arterial phase and early contrast washout in the subsequent phases with capsular enhancement in the delayed phase. The presence of fat content within the lesion was noticed by signal intensity drop of the lesion in the out phase compared to the in-phase image in which the lesion was bright.

On DWIs: The lesion appeared bright, and became brighter with

increasing the $\mathrm{b}$ value. On the ADC map, the lesion became dark. Findings denote restricted diffusion.

Mean ADC value: $0.90 \times 10^{-3} \mathrm{~mm}^{2} / \mathrm{sec}$.

Suggested MR diagnosis: Fat containing Hepatocellular Carcinoma. 


\section{CASE 1:}
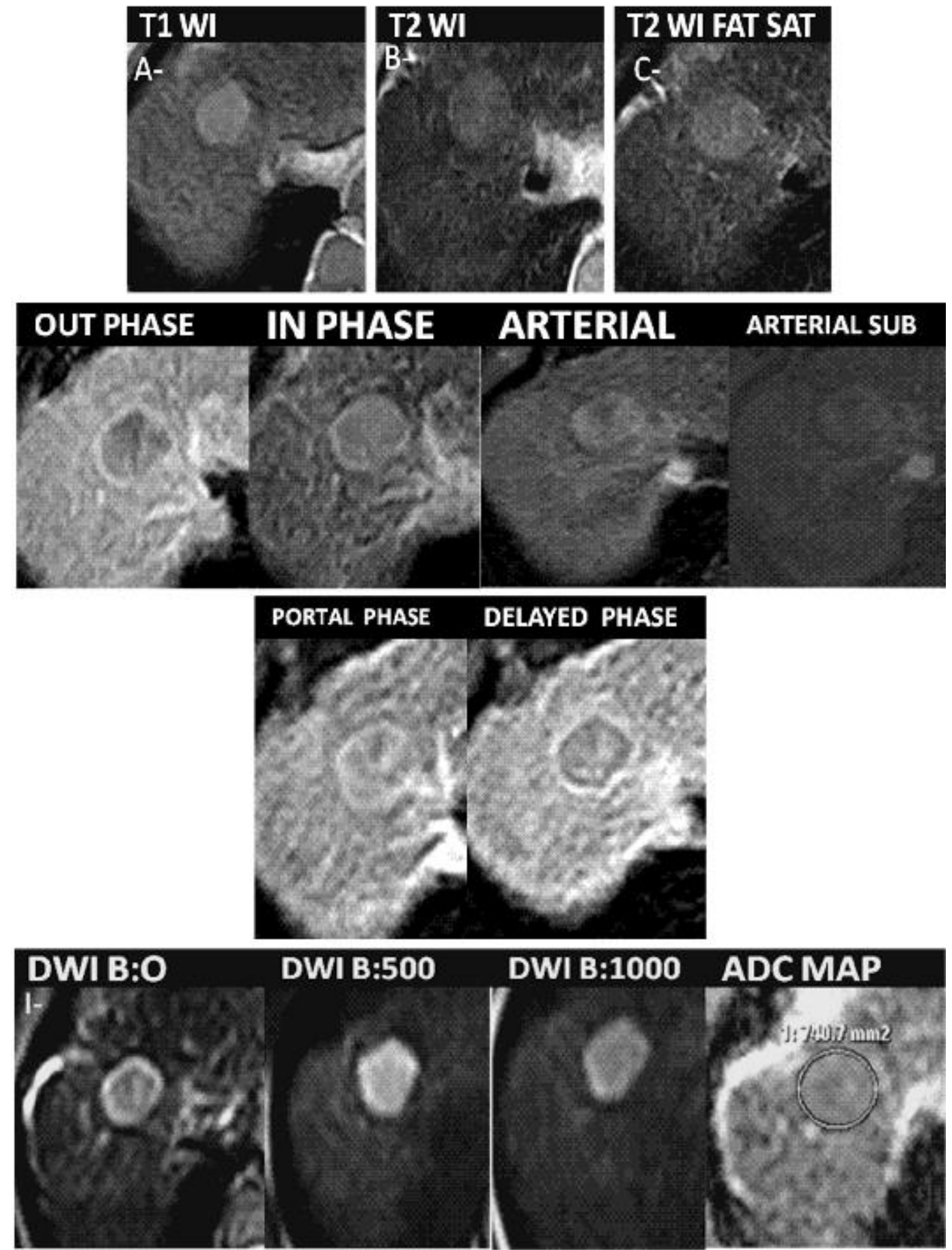

Fig. (1): (A) Axial unenhanced T1W image: high signal intensity lesion within the right hepatic lobe. (B), (C) Axial T2W \& Axial T2 fat sat images: mildly increased signal intensity lesion. (D), (E) Axial out-phase \& Axial in-phase images: showed a signal drop of the lesion in the out-phase compared to the in-phase. (F) The axial gadoliniumenhanced arterial-phase image showed mild enhancement of the lesion as evident by the arterial subtraction image. $\{(\mathrm{G}),(\mathrm{H})\}$ Axial portal gadolinium-enhanced \& Axial delayed gadolinium-enhanced images showed washout of the lesion with capsular enhancement. (I) DWI (b 0,500,1000) showed the lesion to have increased signal intensity owing to restricted diffusion. Axial ADC map showed a lesion of mildly decreased signal intensity proving that the high signal intensity on DWI was notT2 shine-through effect, but a truly restricted diffusion. 


\section{CASE 2:}

Clinical history: A 59 year-old female patient, known case of breast carcinoma underwent a mastectomy and followed by chemotherapy.

On follow up the US for metastatic workup the liver showed multiple hypoechoic focal lesions.

On MRI examination: The liver showed multiple innumerable focal lesions eliciting hypo-intense signals in T1 WIs and hyper-intense signals on T2 WIs.

On DWIs: The lesions appeared bright. On increasing the $b$ value the center of most lesions became dark while the periphery became brighter. On the ADC map, the bright periphery became dark (low ADC value) while the dark center became bright (high ADC value). These findings denote restricted diffusion at the periphery of the lesions while the center of the lesions showed facilitated diffusion of intrinsic tissue necrosis.

ADC values of 10 lesions were measured; $0.86 \times 10^{-3} \mathrm{~mm}^{2} / \mathrm{sec} 1.08 \times 10^{-3} \mathrm{~mm}^{2} / \mathrm{sec} 1.06 \times 10^{-3} \mathrm{~mm}^{2} / \mathrm{sec} 1.19 \times 10^{-3}$ $\mathrm{mm}^{2} / \mathrm{sec} 1.26 \times 10^{-3} \mathrm{~mm}^{2} / \mathrm{sec} 0.95 \times 10^{-3} \mathrm{~mm}^{2} / \mathrm{sec} 1.01 \times 10^{-3} \mathrm{~mm}^{2} / \mathrm{sec} 0.92 \times 10^{-3} \mathrm{~mm}^{2} / \mathrm{sec} 1.24 \times 10^{-3} \mathrm{~mm}^{2} / \mathrm{sec} 1.02 \times 10^{-3}$ $\mathrm{mm}^{2} / \mathrm{sec}$

Mean ADC value; $1.04 \times 10^{-3} \mathrm{~mm}^{2} / \mathrm{sec}$

Suggested MR diagnosis: Breast carcinoma with metastatic liver deposits
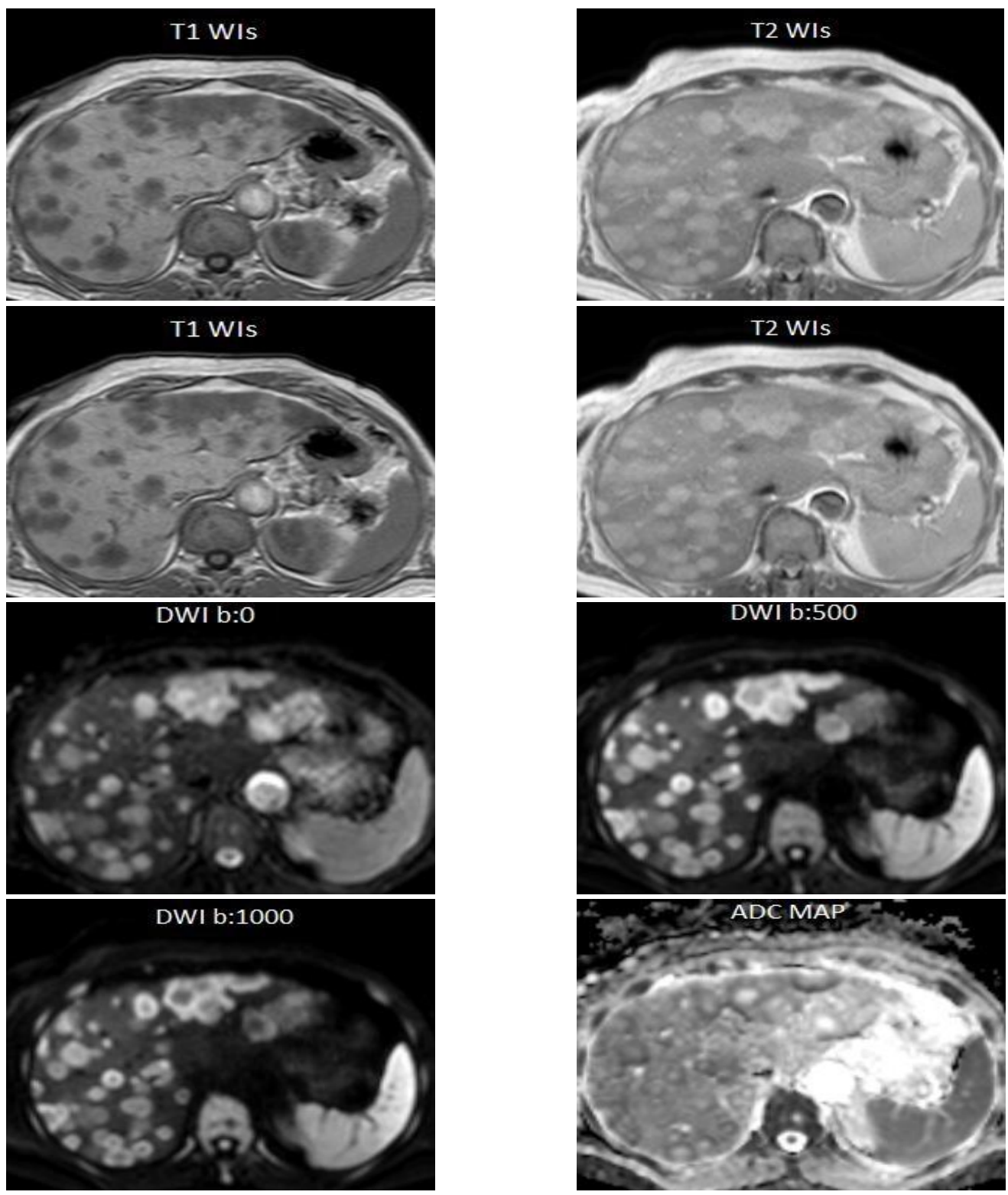

Fig. (2): Multiple innumerable focal lesions eliciting hypo-intense signals in T1WIs and hyperintense signals on T2WIs. On increasing the $b$ value the center of most lesions became dark while the periphery became brighter. On the ADC map, the bright periphery became dark while the dark center became bright. 


\section{DISCUSSION}

The liver is an organ in which various benign or malignant, primary or secondary masses can be detected. Today, focal masses are diagnosed using ultrasonography and/or computed tomography. Additionally, magnetic resonance imaging is preferred when further characterization of these masses is needed. MRI has many advantages (e.g., high contrast resolution, the ability to obtain images in any plane, lack of ionizing radiation, and the safety of using particulate contrast media rather than those containing iodine) that make it a favored modality ${ }^{(7)}$.

DW-MRI provides unique insight into tissue cellularity, tissue organization, the integrity of cells and membranes, as well as the tortuosity of the extracellular space, which can help detect malignant diseases, and for distinguishing tumor tissues from non-tumor tissues ${ }^{(8)}$.

The current study was conducted including thirty patients, 24 males, and 6 females, with age ranging from $40-83$ years and mean age of 60.2 years.

In our study diffusion images were obtained before intra-venous contrast administration which was also mentioned ${ }^{\left({ }^{9}\right)}$ which stated that DW MR imaging of the liver is usually performed before contrast material administration, although performing DW MR imaging after the administration of contrast did not appear to significantly affect ADC calculations ${ }^{(8)}$.

In our study, three different $b$ values were conducted which was in line with the study performed (10)

This study was conducted with high $b$ value $\left(500 \& 1000 \mathrm{sec} / \mathrm{mm}^{2}\right)$ to overcome the effect of capillary perfusion and water diffusion in extracellular extravascular space, as high $b$ value will result in the reduction of signal from moving protons in the bile ducts, cysts, vessels, and fluid in the bowel. This will result in an increased contrast between the lesion and liver. Furthermore, the differences in the relative contrast ratio between malignant and benign lesions were increased with a high $b$ value. This was similar to the $b$ value used in studies carried by ${ }^{(11-13)}$.

All cystic lesions showed facilitated diffusion, where they showed a reduction of signal intensity on increasing the b-values, and those which didn't show the reduction of the signal showed high signal on ADC map, which also reflects facilitated diffusion. On the other hand, all solid lesions showed restricted diffusion evidenced by the increased signal on increasing the $b$ values and low signal on ADC maps.

\section{CONCLUSION}

DW MR imaging has the potential to be a reasonable alternative technique to contrast-enhanced imaging.

Disclosure: The authors report no conflicts of interest in this work.

Source of funding: by self.

\section{REFERENCES}

1. Nijalingappa M, Naveen S (2015): Role of DiffusionWeighted Magnetic Resonance Imaging in Focal Liver Lesions. Journal of Dental and Medical Sciences, 14: 1022.

2. Elbarbary A, Elahwal H, Elashwah M (2015): Role of Diffusion-Weighted Magnetic Resonance Imaging in the evaluation of hepatic focal lesions. The Egyptian Journal of Radiology and Nuclear Medicine, 46:325334.

3. Cosmin C, Liliana C, Diana- Ioana F et al. (2015): Diffusion-Weighted Magnetic Resonance Imaging for the Classification of Focal Liver Lesions as Benign or Malignant. J Gastrointestin Liver Dis., 24(3): 309-317.

4. Zhao X, Zhou S, Wang D et al. (2015): Differential Diagnosis of Malignant Biliary Tract Cancer from Benign Tissues using Apparent Diffusion Coefficient Measurements with Diffusion-Weighted Imaging in Asians. Asian Pacific Journal of Cancer Prevention, 16:6135-6140.

5. Patil S, Sethi M, Kakar S (2014): Morphological Study of Human Liver and its Surgical Importance. International Journal of Anatomy and Research, Int J Anat Res., 2(2): 310-314.

6. Rutkauskas S, Gedrimas V, Pundzius J et al. (2006): Clinical and anatomical basis for the classification of the structural parts of the liver. Medicina (Kaunas), 42(2): 98-106.

7. Demir Öİ, Obuz F, Sağol Ö et al. (2007): Contribution of diffusion-weighted MRI to the differential diagnosis of hepatic masses. Diagn Interv Radiol., 13:81-8.

8. Thoeny HC, De Keyzer F (2007): Effect of vascular targeting agent in rat tumor model: dynamic contrastenhanced versus diffusion-weighted MR imaging. Radiology, 237:492-499.

9. Taouli B, Dow-Mu K (2010): Diffusion-weighted MR imaging of the liver Radiology, 254 (1): 202-209.

10. Qayyum A (2009): Diffusion-weighted Imaging in the Abdomen and Pelvis: Concepts and Applications. Radio Graphics, 29:1797- 1810.

11. Parikh T, Drew SJ, Lee VS et al. (2008): Focal liver lesion detection and characterization with diffusionweighted MR imaging: comparison with standard breath-hold T2-weighted imaging. Radiology, 246:812822.

12. Koike N, Cho A, Nasu $K$ et al. (2009): Role of diffusion-weighted magnetic resonance imaging in the differential diagnosis of focal hepatic lesions World $\mathbf{J}$ Gastroenterol., 15: 5805-5812.

13. Hosny IA, Janshir J (2010): Diffusion MRI of focal liver lesions Pak J Radiol., 20: 1-7. 